\title{
Analysis of Periodicities in Surface Topography of Cold rolled sheets Using Data Captured by Camera System
}

\author{
Jaromir Zavadil ${ }^{1}$, Josef Strom Bartunek ${ }^{2}$, David Fojtik ${ }^{1}$ \\ ${ }^{I}$ Department of Control Systems and Instrumentation, Faculty of Mechanical Engineering, VSB - Technical University of \\ Ostrava, 17. listopadu 2172/15, 70800 Ostrava-Poruba, Czech Republic, jaromir.zavadil@vsb.cz \\ ${ }^{2}$ Department of Applied Signal Processing, Blekinge Institute of Technology, SE-371 79 Karlskrona, Sweden
}

\begin{abstract}
A method for surface analysis of cold rolled sheets is proposed in this paper. The approach is based on a low-cost specially built camera system followed by spectral analysis of the data captured from metal surfaces. The focus is on the changes in the surface topography caused by cold rolling with emphasis towards periodicities in the processed surface. Angular profile of the spectrum is calculated and used to display periodicities in surface topography and show their direction. The results obtained by using the proposed system were compared with results obtained from the optical profilometer MicroProf FRT. The experiments show that cold rolling creates marks on the surface of the material, which represent periodicities that can be effectively detected by the proposed method and camera system. Even though the camera system is not able to measure precise surface roughness, it is able to detect periodicities and the results of spectral analysis are comparable with the results from the optical profilometer.
\end{abstract}

Keywords: Surface analysis, cold rolling, optical profilometer, camera system, periodicities.

\section{INTRODUCTION}

Analysis of surfaces is an important task in many areas of industry where surface quality has an important role. In these areas it is essential to set such machining or forming parameters to obtain the best quality of the processed surface. Therefore, the focus is on innovative methods for the analysis of surfaces of cold rolled sheets. For example, Dong Xu et al. performed an experimental study of the digital speckle patterns generated by rough surfaces illuminated by a laser [1], Sheng-He Chen et al. proposed an adaptive regression smoothing filtering method for on-line surface roughness detection of cold rolled strip steel [2], R. Ahmed and M. P. F. Sutcliffe described identification of surface features on coldrolled stainless steel strip to automatically detect pits and roll marks that can be observed in optical or SEM micrographs [3]. Szarková et al. proposed a method of surface evaluation of steel strips formed by longitudinal cold rolling with focus on the impact of grain size of material with a rolling reduction and rolling force [4]. Valicek at al. proposed a method of maintaining the required values of surface roughness and prediction of technological conditions for cold rolling [5].

Parameters for surface metrology are still developing with the current trend of transition from two-dimensional profile parameters to three-dimensional spatial parameters using a large variety of methods for measurements of surface roughness or topography. These can be divided into two categories: contact and contactless methods, each having some advantages and disadvantages [6]. Contactless methods are usually based on optical spectrometers.

Currently, the most frequently used parameters for engineering surface evaluation are two-dimensional (profile) height parameters, for example: arithmetical mean height $\mathrm{Ra}$, which is the arithmetic average of the absolute values of the profile heights along the evaluation length; root mean square deviation $\mathrm{Rq}$, which is the root mean square average of the profile heights along the evaluation length; maximum height of the profile $\mathrm{Rz}$, which is the absolute vertical distance between the maximum profile peak height and the maximum profile valley depth along the evaluation length.

Besides these, there are many other parameters included in ISO 4287 [7]. These parameters are usually measured by contact profilometers. Newer three-dimensional (aerial) height parameters are introduced in ISO 25178 [8], for example $\mathrm{Sa}, \mathrm{Sq}, \mathrm{Rz}$, which are extensions of $\mathrm{R}$ parameters to a surface:

$$
\begin{gathered}
S a=\frac{1}{M N} \sum_{k=0}^{M-1} \sum_{i=0}^{N-1}\left|z\left(x_{k}, y_{i}\right)\right| \\
S q=\sqrt{\frac{1}{M N} \sum_{k=0}^{M-1} \sum_{i=0}^{N-1}\left[z\left(x_{k}, y_{i}\right)\right]^{2}}
\end{gathered}
$$




$$
S z=S p+S v
$$

where $S p$ is the largest peak height and $S v$ is the largest valley depth value within the evaluation area.

There are also other height parameters. These aerial height parameters allow better representation of the surface topography. New technical specification ISO 25178 introduced also completely new parameters, for example auto-correlation length and texture aspect ratio.

All the above-mentioned parameters require high precision measurement tools which are usually very expensive. In addition, measurement speed of these tools is very slow, which makes them unsuitable for any real time quality inspections. In current praxis, only some samples are selected and taken to the laboratory for analysis which is time consuming. It is, therefore, the main purpose of this paper to propose a contactless measurement method for surface evaluation that is of low cost and fast so it could be used in real time during the machining or forming processes. Results of these measurements could be used for online evaluation of the resulting surface and monitoring of the roller in real operation plant. The cold rolling process gives rise to marks on the surface of the material which are periodically repeated. Thus, the proposed method uses spectral analysis on images captured with a specially built camera system to analyze these periodicities.

\section{SUBJECT \& METHODS}

Deep-drawing steel sheets, type KOHAL 697 from U. S. Steel Kosice, were used for experiments described in this paper. Samples were created by cold rolling of several strips with dimensions $150 \times 31 \times 2.52 \mathrm{~mm}$. Each sample was rolled on the rolling stand DUO $210 \mathrm{SVa}$ with $v_{\text {roll }}=1 \mathrm{~ms}^{-1}$. Ten samples (S1-S10) were created by rolling with different thickness reduction $\Delta h$ and one unprocessed sample (S0) was kept without rolling as reference. Parameters of these samples can be found in Table 1., where $h 0$ is the thickness of the sample before rolling and $h 1$ is thickness of the sample after rolling.

Table 1. Reductions $\Delta h$ for each sample.

\begin{tabular}{|c|c|c|c|}
\hline $\begin{array}{c}\text { Sample } \\
\text { label }\end{array}$ & $\begin{array}{c}\boldsymbol{h} \mathbf{( \mathbf { m m } )} \\
\mathbf{( m )}\end{array}$ & $\begin{array}{c}\boldsymbol{h 1} \\
(\mathbf{m m})\end{array}$ & $\begin{array}{c}\Delta \boldsymbol{h} \\
(\mathbf{m m})\end{array}$ \\
\hline S0 & 2.50 & - & - \\
\hline S1 & 2.50 & 2.25 & 0.25 \\
\hline S2 & 2.50 & 1.94 & 0.56 \\
\hline S3 & 2.49 & 1.74 & 0.75 \\
\hline S4 & 2.49 & 1.54 & 0.95 \\
\hline S5 & 2.51 & 1.31 & 1.20 \\
\hline S6 & 2.46 & 1.11 & 1.35 \\
\hline S7 & 2.48 & 0.91 & 1.57 \\
\hline S8 & 2.48 & 0.83 & 1.65 \\
\hline S9 & 2.47 & 0.75 & 1.72 \\
\hline S10 & 2.49 & 0.51 & 1.98 \\
\hline
\end{tabular}

Example of an image with unprocessed surface before rolling can be seen in Fig.1. and an image of a surface after rolling can be seen in Fig.2.

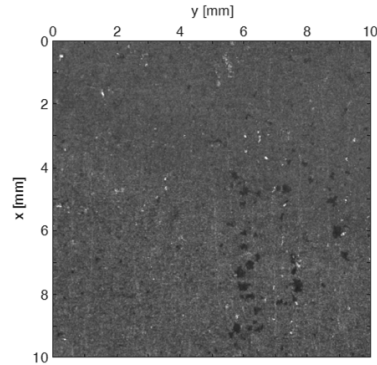

a)

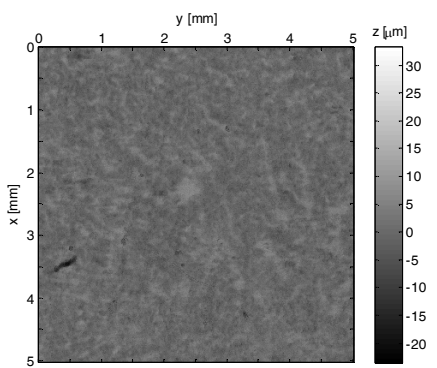

b)
Fig.1. Image of the surface without rolling. a) - acquired using the camera system described in this paper b) - acquired using the profilometer MicroProf FRT.

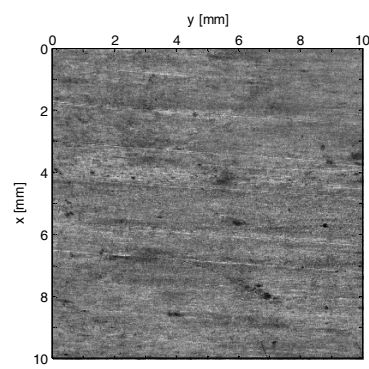

a)

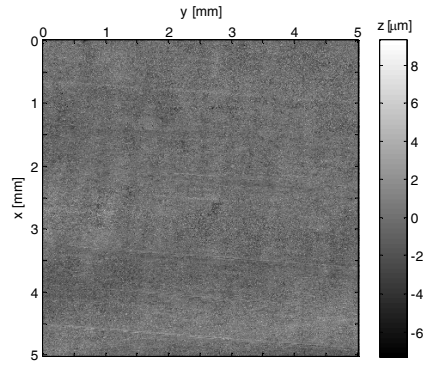

b)
Fig.2. Image of the surface after rolling a) - acquired using the camera system described in this paper b) - acquired using the profilometer MicroProf FRT.

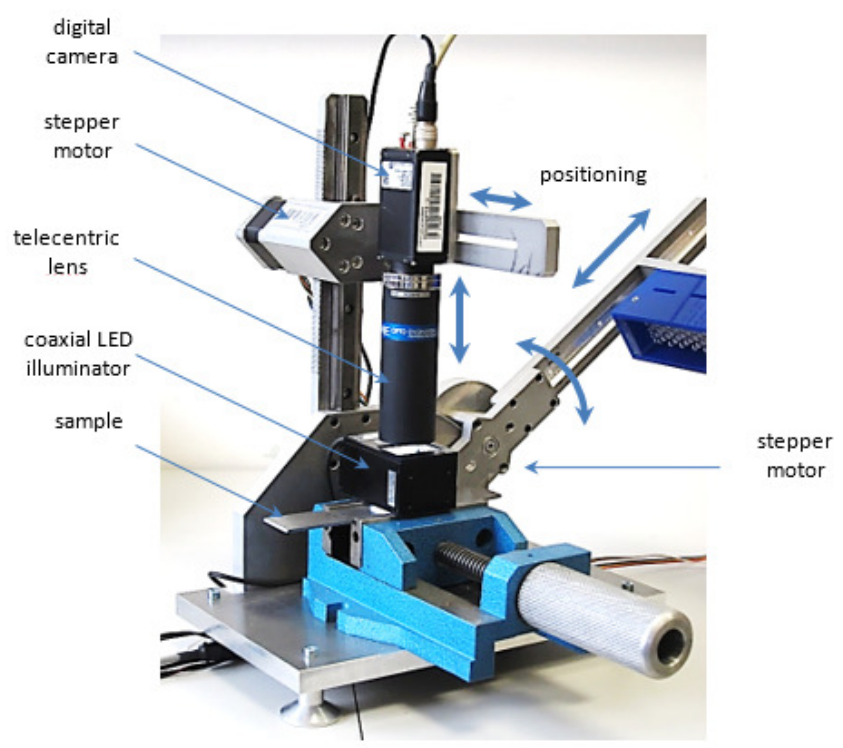

Fig.3. Proposed camera system setup.

Special camera system has been built for capturing the surface topography. This system is composed of the area scan digital camera Basler Ace acA1600-20gm and bilateral telecentric lens Opto Engineering TC 2316 mounted on a metal frame along with a controllable coaxial LED illuminator, see Fig.3. Stepper motors Leadshine Technology $42 \mathrm{HS} 03$ are used for precise positioning of the camera. Maximum frame rate of the camera is $20 \mathrm{fps}$. 
The camera system is controlled through M415B drivers and Arduino Due via user interface created in C\#. Created software application is used also for image acquisition. The flowchart of the controlling system is shown in Fig.4.

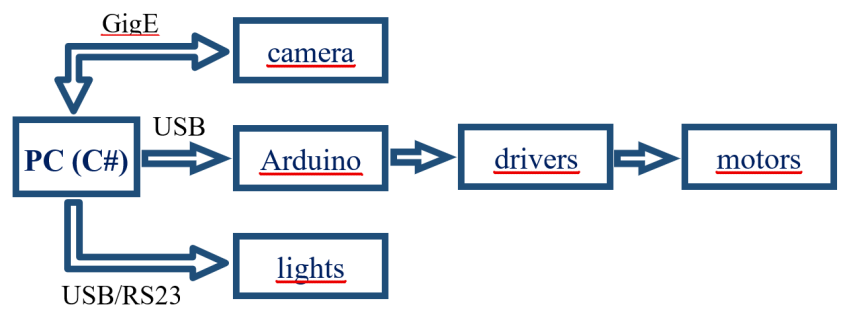

Fig.4. Flowchart of the controlling of the camera system.

Field of view with used lenses was $13.5 \times 10.2 \mathrm{~mm}(1628 \mathrm{x}$ 1236 points were measured). Area of size $1236 \times 1236$ points $(10.2 \times 10.2 \mathrm{~mm})$ has been selected and consequently processed using MATLAB. Application created in MATLAB transforms the acquired data into frequency domain and calculates the angular spectrum as described below. For comparison, the surface topography of the samples has been also measured using the optical profilometer MicroProf FRT. The area measured by the profilometer was 5 x $5 \mathrm{~mm} \mathrm{(1024}$ x 1024 points).

For aerial measurements (three-dimensional), each sample of data measured is represented as a matrix of dimension $M \mathrm{x}$ $N$ and is expressed as

$$
f(x, y)=\left[\begin{array}{cccc}
f(0,0) & f(0,1) & \cdots & f(0, N-1) \\
f(1,0) & f(1,1) & \cdots & f(1, N-1) \\
\vdots & \vdots & \cdots & \vdots \\
f(M-1,0) & f(M-1,1) & \cdots & f(M-1, N-1)
\end{array}\right]
$$

where $x=0,1,2, \ldots, M-1$ and $y=0,1,2, \ldots, N-1$ are the coordinates and $\mathrm{f}(x, y)$ consists of the topographic value of the captured surface in grey scale.

Analysis of the captured surface is performed using 2D DFT as follows

$$
F(u, v)=\frac{1}{M N} \sum_{x=0}^{M-1} \sum_{y=0}^{N-1} f(x, y) e^{-j 2 \pi(u x / M+v y / N)}
$$

where $u=0,1,2, \ldots, M-1$ and $v=0,1,2, \ldots, N-1$ are the coordinates in the spectrum. The resulting $M \mathrm{x} N$ matrix contains the 2D frequency spectrum of the surface. Analysis of the result of the transform is based on computing the spectrum (magnitude of $\mathrm{F}(u, v)$.

$$
|F(u, v)|=\sqrt{R^{2}(u, v)+I^{2}(u, v)}
$$

In Fig.5. the spectrum of the unprocessed surface shown in Fig.1. is observed. In Fig.6. the spectrum of the processed surface shown in Fig.2. is observed. Rolling process introduces noticeable marks on the surface. These marks have specific direction which can be clearly observed in its spectrum. It should be noted that despite the higher resolution of the camera system, there are less points per $1 \mathrm{~mm}$ taken comparing to data from the optical profilometer due to a larger field of view of the camera system.

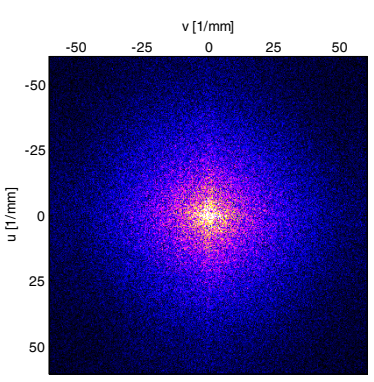

a)

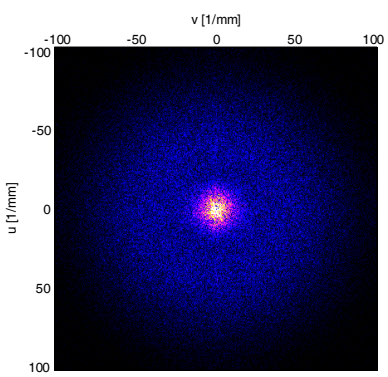

b)
Fig.5. Magnitude spectrum of unprocessed surface a) - data obtained from the proposed camera system b) - data obtained from the profilometer MicroProf FRT.

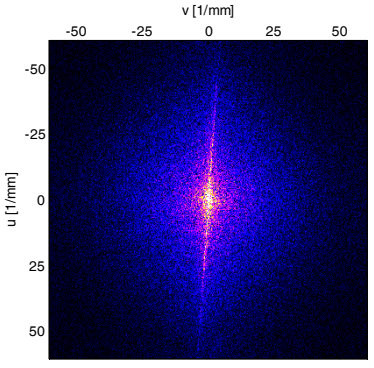

a)

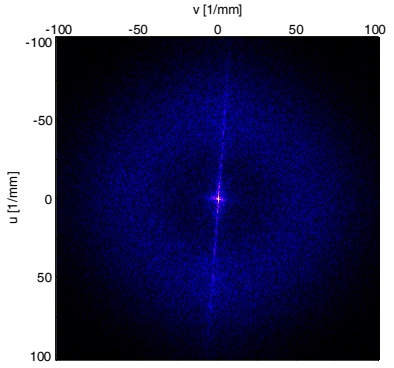

b)
Fig.6. Magnitude spectrum of surface after rolling a) - data obtained from the proposed camera system b) - data obtained from the profilometer MicroProf FRT.

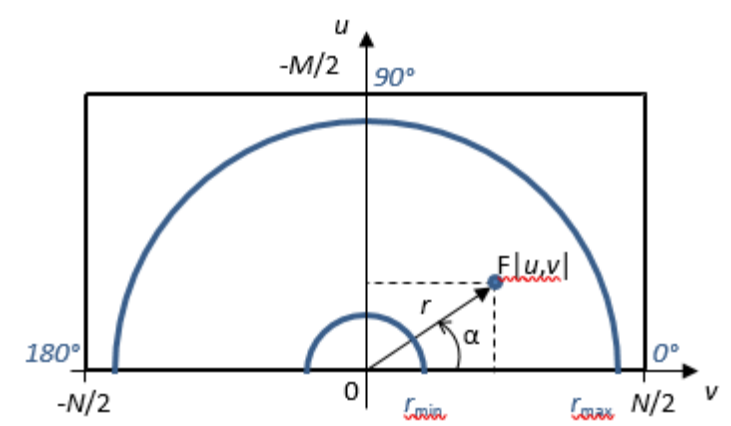

Fig.7. Conversion of magnitude spectrum to polar coordinates.

The two-dimensional magnitude spectrum is converted from Cartesian to polar coordinates to analyze surface anisotropy and texture direction. The conversion process is illustrated in Fig.7. For each point in the spectral map $|\mathrm{F}(u, v)|$ the angle $(\alpha)$ is calculated along with distance $(r)$ of this point from the center of the frequency rectangle. Then the amplitudes for all points with the same angle are added to create an angular profile of the magnitude spectrum. The distance $(r)$ is used to select a range of wavelength for amplitude adding $\left(r_{\min }\right.$ and $\left.r_{\max }\right)$. 
The calculation of angle $(\alpha)$ and distance from center $(r)$ is done based on the following formulas:

$$
\alpha=\left\{\begin{array}{ccc}
\operatorname{atan} \frac{u}{v} & \text { for } & v>0, u>0 \\
90^{\circ} & \text { for } & v=0, u>0 \\
\operatorname{atan} \frac{u}{v}+180^{\circ} & \text { for } & v<0, u>0 \\
180^{\circ} & \text { for } & v<0, u=0
\end{array} \quad r=\sqrt{u^{2}+v^{2}}\right.
$$

\section{Results}

As mentioned at the beginning of this paper, unlike the traditional measurements of the surface roughness, the focus of this approach is on the changes in periodicities in the surface topography caused by cold rolling.

To obtain the same frequency range for both measurements using the proposed camera system and the optical profilometer, the parameters $r_{\min }$ and $r_{\max }$ were set to $r_{\min }=4$ and $r_{\max }=204$ for images acquired by the proposed camera system, and $r_{\min }=1$ and $r_{\max }=100$ for images acquired by the optical profilometer. This is due to the different image resolution of the data captured by each device. Selected values of $r_{\min }$ and $r_{\max }$ parameters correspond to frequency range $f_{\min }=0.2 \mathrm{~mm}^{-1}$ and $f_{\max }=10 \mathrm{~mm}^{-1}$ for both measurements.

Fig.8. and Fig.9. show angular profile of the spectrum calculated for all measurements. It can be observed that despite the different capturing devices used for each measurement, both types of data show the periodicities created on the surface during the rolling process. The angular spectrum of the unprocessed surface without rolling is homogeneous in all directions while the angular spectra of the rolled samples revealed periodicities created during the rolling process.

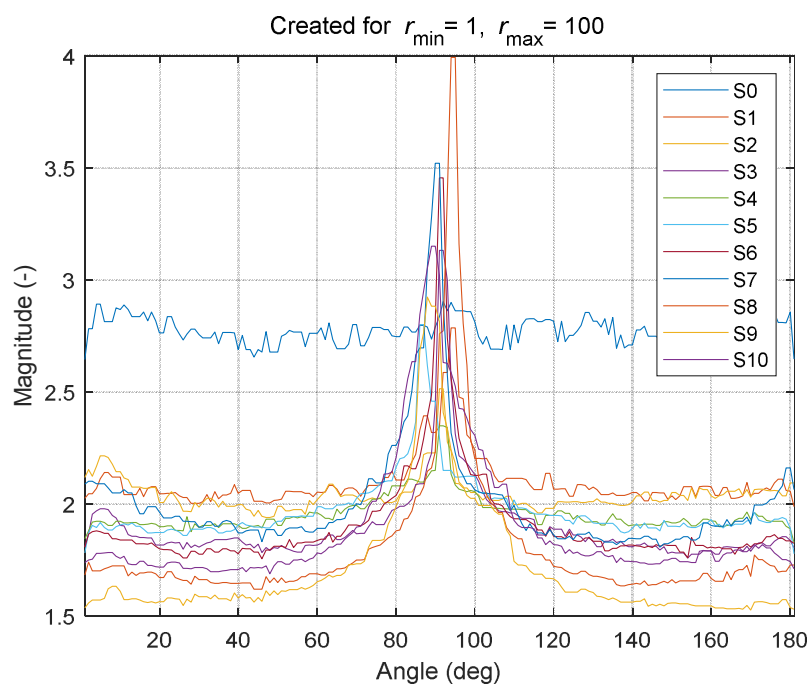

Fig.8. Angular spectrum from images obtained from the profilometer MicroProf FRT.

It should be noted that the topography of the roller is transferred to the rolled sheet, once the reduction rate is over
$10 \%$. That is why there are little differences between samples S2-S10. Future extension to these experiments will be rolling of the samples by the rollers with different surface texture characteristic.

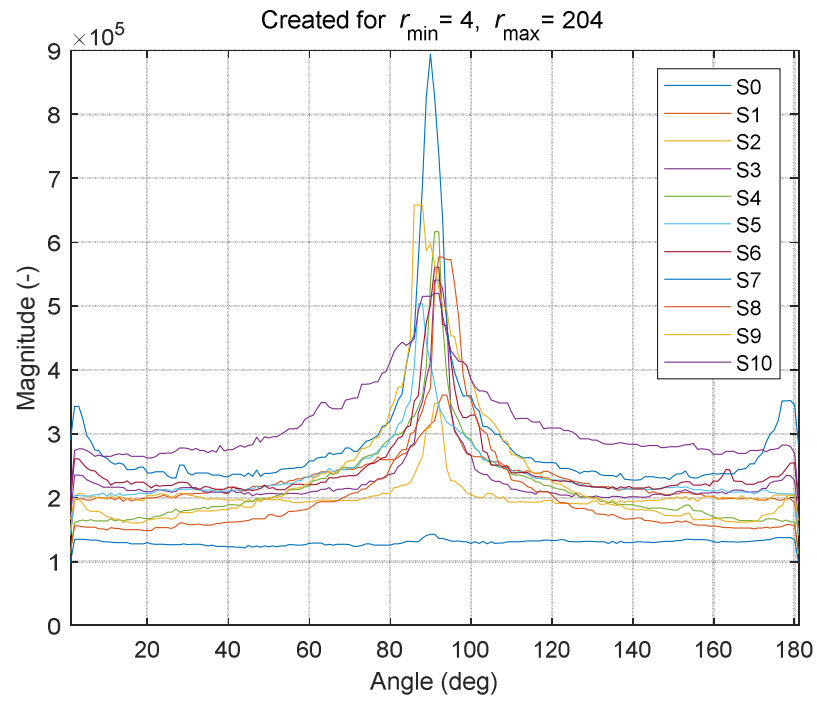

Fig.9. Angular spectrum from images obtained from the proposed camera system.

One of the biggest advantages of the proposed camera system is the speed of measurement. This camera system is able to capture the whole field of view at once where the array of $10.2 \times 10.2 \mathrm{~mm}$ was captured in about 1 second, while the same measurement using the optical profilometer took about 30 minutes.

\section{CONCLUSIONS}

This paper presented a novel approach to the surface analysis of cold rolled sheets using a camera system and spectral analysis of data. Unlike traditional approaches, this approach is based on the processing of image obtained from an ordinary camera. Angular profile of the spectrum is calculated and used to display periodicities in surface topography and show their direction. Ten samples of cold rolled sheets with different reductions were used for the analysis and their surface was measured using a camera system assembled for the purpose of this experiment. Results obtained by this measurement were then compared with measurements using the optical profilometer MicroProf FRT.

Experiments proved that the new camera system can be efficiently used for the analysis of periodicities in a surface topography of cold rolled sheets. Cold rolling creates marks on the surface of the material, which represent periodicities that can be effectively detected by this camera system. Even though the camera system is not able to measure precise surface roughness, it is able to detect periodicities in a much faster manner than the optical profilometer and could be therefore used during surface rolling. Results of these measurements could be used for online evaluation of the resulting surface and monitoring of the roller in real operation plant. 
Maximum frame rate of the camera used for the experiments is 20fps which would be sufficient for applications in operation plant with a speed of the belt movement approximately up to $0.1 \mathrm{~ms}^{-1}$. However, there are cameras with frame rates of hundreds or thousands fps so the camera can be selected based on the speed of the belt movement. Also, a combination of a camera and an lens with a larger field of view could be selected. The larger view of field allows capturing the larger surface array at once.

Next extension of this work will be quantification of the periodicities of the surface topography and experiments with the rollers with different surface texture characteristics.

\section{ACKNOWLEDGMENT}

This work was supported by the European Regional Development Fund in the Research Centre of Advanced Mechatronic Systems project, CZ.02.1.01/0.0/0.0/16 019/0000867 within the Operational Programme Research, Development and Education and the project SP2019/51 Applied Research in the Area of Machine and Process Control supported by the Ministry of Education, Youth and Sports.

\section{REFERENCES}

[1] Xu, D., Yang, Q., Dong, F., Krishnaswamy, S. (2018). Evaluation of surface roughness of a machined metal surface based on laser speckle pattern. The Journal of Engineering, 2018 (9), 773-778.

[2] Chen, S.-H., Wang, J.-G., Gu, T.-Q. (2018). Online surface roughness detection of cold-rolled strip steel based on adaptive regression smooth filtering. In 37th Chinese Control Conference (CCC). IEEE, 8325-8329.
[3] Ahmed, R., Sutcliffe, M.P.F. (2000). Identification of surface features on cold-rolled stainless steel strip. Wear, 224 (1-2), 60-70.

[4] Szarková, V., Valíček, J., Vlado, M., Harničárová, M., Rokosz, K., Lupták, M., Samardžić, I., Kozak, D., Hloch, S. (2013). Influence of longitudinal cold rolling on the surface topography of low carbon structural steel. Technical Gazette, 20 (4), 705-709.

[5] Valíček, J., Harničárová, M., Kušnerová, M., Zavadil, J., Grznárik, R. (2014). Method of maintaining the required values of surface roughness and prediction of technological conditions for cold sheet rolling. Measurement Science Review, 14 (3), 144-151.

[6] Piska, M., Metelkova, J. (2014). On the comparison of contact and non-contact evaluations of a machined surface. MM Science Journal, 2014 (2), 476-480.

[7] International Organization for Standardization. (1997). Geometrical Product Specifications (GPS) -- Surface texture: Profile method -- Terms, definitions and surface texture parameters. ISO 4287:1997.

[8] International Organization for Standardization. (2012). Geometrical product specifications (GPS) -- Surface texture: Areal -- Part 2: Terms, definitions and surface texture parameters. ISO 25178-2:2012

Received January 24, 2020 Accepted July 06, 2020 